\title{
Remote System Setup Using Large-Scale Field Programmable Analog Arrays (FPAA) to Enabling Wide Accessibility of Configurable Devices
}

\author{
Jennifer Hasler*, Sahil Shah, Sihwan Kim, Ishan Kumal Lal and Michelle Collins \\ Electrical and Computer Engineering (ECE), Georgia Institute of Technology, Atlanta, GA 30332-250, USA; \\ scorpiojackson21@gmail.com (S.S.); k.sihwan@gmail.com (S.K.); ishanlal@gatech.edu (I.K.L.); \\ jenniolson@gmail.com (M.C.) \\ * Correspondence: jennifer.hasler@ece.gatech.edu; Tel.: +1-404-894-2944; Fax: +1-404-894-4641 \\ Academic Editor: Tony Tae-Hyoung Kim \\ Received: 4 March 2016; Accepted: 14 July 2016; Published: 28 July 2016
}

\begin{abstract}
We present a novel remote test system, an integrated remote testing system requiring minimal technology support overhead, enabled by configurable analog-digital Integrated Circuits (IC) to create a simple interface for a wide range of experiments. Our remote test system requires no additional setup, resulting both from using highly configurable devices, as well as from the advancement of straight-forward digital interfaces (i.e., USB) for the resulting experimental system. The system overhead requirements require simple email handling, available over almost all network systems with no additional requirements. The system is empowered through large-scale Field Programmable Analog Array (FPAA) devices and Baseline Tool Framework (BTF), where we present a range of experimentally measured examples illustrating the range of user interfacing available for the remote user.
\end{abstract}

Keywords: FPAA; remote test system; SoC FPAA; Design tools

This paper discusses a novel remote test system, enabled by configurable analog-digital Integrated Circuits (IC) to create a simple interface for a wide range of experiments; a wide range of previous remote test systems have to spend considerable time developing their hand-tailored configurable system [1-6]. These systems are often specialized for a particular experiment, and the infrastructure might not resemble the setup with hardware in hand. Students have to learn (and debug) yet another system, increasing the barrier to use.

Figure 1 illustrates the remote test system requiring no additional setup, other than the experimental system, and simple email handling, available over almost all network systems. Independent of distance, the system enables users anywhere with an internet connection that is sufficient to send and receive email, providing opportunities both in academic as well as research and industrial applications. This approach minimizes computer support setup and maintenance, relieving the pressure on overworked computer support staff, particularly in cost-conscious academic environments, as they try to keep pace to maintain a larger number of computing systems. Those creating the system do not require any web hosting, as well as any web-based executable functions requiring numerous signoffs and security checks for any code iteration. The system only requires a simple unmanaged WiFi or cable connection, even including university guest WiFi or WiFi in a coffee shop. Computer administrative staff will have no further knowledge of this system compared to a guest user on an unsecured WiFi. One can imagine extending this concept to a number of remote sensor nodes. 


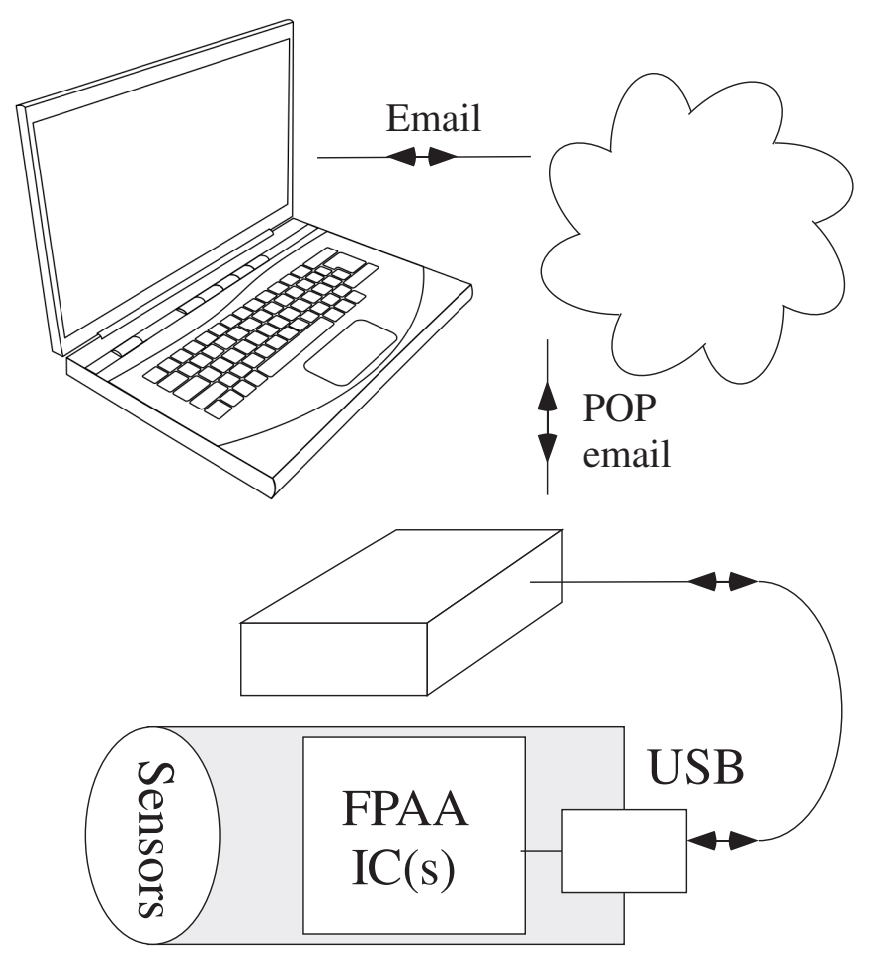

Figure 1. Remote test system based on large-scale Field Programmable Analog Array (FPAA) devices that can be used within our current framework of high-level, open-source Xcos/Scilab tools. With a single button click in the graphical tool, the system will email the resulting targeting code for the FPAA device to a server location to be picked up by the remote system, that compiles, runs, and then emails back the target results.

The remote test infrastructure is enabled through a single external digital interface to an analog-digital programmable and configurable IC system, empowered using large-scale Field Programmable Analog Array (FPAA) device(s) [7]. This approach gives a simple digital peripheral using a standard interface (i.e., USB), enabling a small Internet of Things (IoT) block interfaced through an email system to an open-source design/control tool. Our open-source tool platform empowers the user to do seamless low-power analog-digital co-design in a single environment [8]. The resulting controlling device, whether it be directly connected through this digital port (i.e., phone or tablet) or through a network, can be a potentially simple OS enabling all features on the resulting system, including sensory/actuation devices connected to this remote platform.

POP (Post Office Protocall) email communication requires only a few limited operations, requires simple coding, and requires minimal web-based computation requirements for operation, particularly for academic environments. Email protocol simplifies individual access while requiring effectively zero administrative support. Other educational remote test systems require the user to take control of the system, usually through a log-in. This remote system approach differs from the area of one-way updating FPGA software, or remote FPGA reconfiguration for a device in the field $[9,10]$.

The following sections present the resulting FPAA based remote test setup including overviewing the remote-tool structure, overviewing the FPAA devices and Baseline Tool Framework (BTF) for a self-contained discussion, presenting multiple system examples, while presenting the range of user interfacing, and expanding the remote user application as well as measurement capability. 


\section{Remote Tool Framework and FPAA Overview}

Figure 2 shows the perspective of our remote testing approach. When one thinks of testing an analog IC, or even a mixed mode IC, one tends to visualize, in the best case, a test setup like Figure 3a. Such systems mostly have a single or a group of analog ICs, some controlled switches, and an infrastructure to connect to a computer to control the entire setup as well as run the testing interface. When looking at a remote testing system, the testing interface layer requires further complication depending on the particular system.

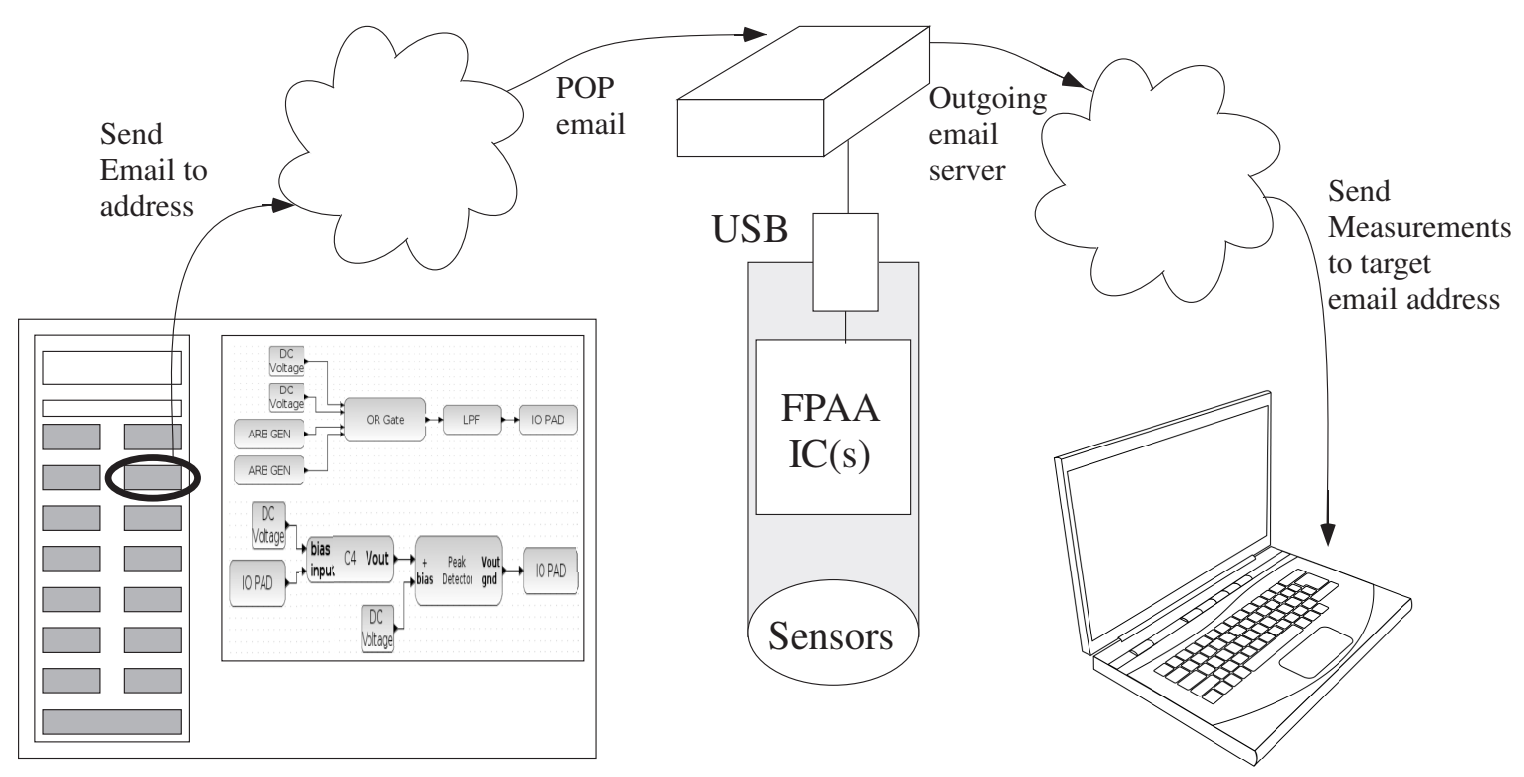

Figure 2. Detailed flow for the remote test system implementation. The design toolset in Xcos/Scilab allows the user to "send email" in addition to "program FPAA". When that option is chosen, the resulting file is sent by email into the cloud. The resulting email is POP-ed off the server, the resulting programming files are extracted and executed, the resulting data measurement is performed on the device, and the results are sent back by email to the original sender. The user can directly use the results in Scilab or any other data analysis program to observe their data as well as complete their analysis. The resulting flow is enabled by having a highly configurable analog/mixed-mode system with a simple digital interface through USB, really enabling the connection as a typical digital peripheral.

In comparison, Figure $3 \mathrm{~b}$ shows the FPAA system used in this paper. The FPAA IC is a full system with a processor, requiring only simple interfacing to the outside world through USB or say SPI ports, appearing to be a standard peripheral to a typical device. Such an approach enables a family of configurable hardware, utilizing a single configurable framework and tool infrastructure to control the resulting device. This difference in configuration provides the opportunity for empowering our remote test system for classroom use, research groups, as well as interested users. Integrating this approach into an existing tool framework keeps compatibility for a range of applications, and not limited to just an academic or research application.

Figure 2 shows the framework for the remote system approach. The resulting structure should be easy to use on both the user and remote server side, requiring minimal user maintenance, using an integrated user tool platform, and having as few location constraints as possible. The tool platform [8], implemented in Xcos/Scilab (an open source clone for MATLAB/Simulink), simulates designs as well as enables experimental measurements after compiling to the same integrated design tool framework. The open-source toolset is setup as an Ubuntu 12.04 Virtual Machine (VM) (available at http:/ / users.ece.gatech.edu/phasler/FPAAtool/index.html) enabling use in classrooms as well as research and development groups. 


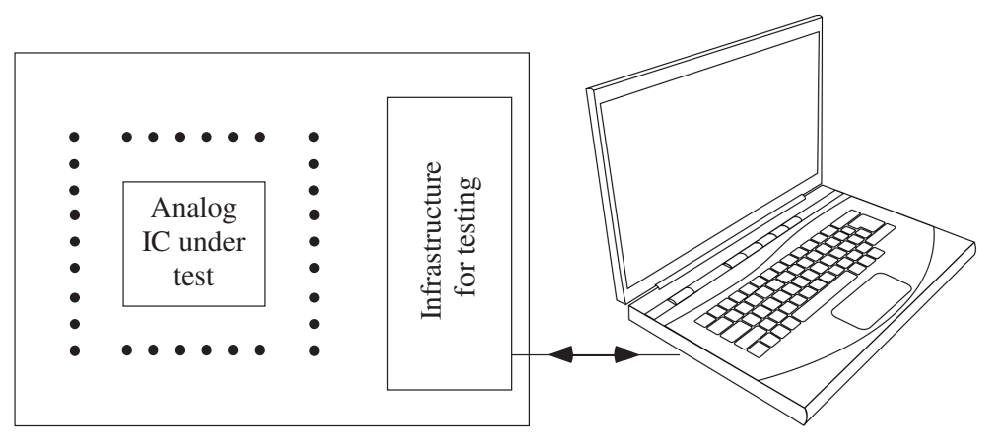

(a)

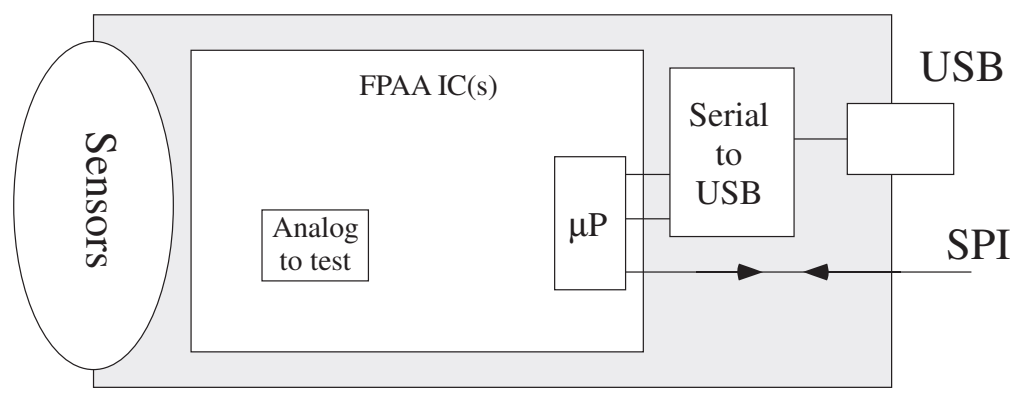

(b)

Figure 3. System perspective using a remote test system to utilize mixed-signal configurable systems. (a) classical approach when considering analog IC design and testing that have an analog component under test, along with all of the required board (or bench) infrastructure required; (b) for these mixed signal ICs, the IC is an entire system, acting as a peripheral through a USB port and/or optional SPI port. In addition, the resulting analog to test, if making a complete parallel to the system in (a), is a small part of the overall available computing infrastructure.

Our modifications to the high level Xcos tools on the user side required extending the GUI interface, as well as updating the (Python) code to enable emailing the resulting compiled FPAA targeting file out from the VM. The user will receive their resulting data to their email location as an attached representation of the measured results; the user can move this data into Scilab or another analysis/plotting tool of their choice. By using an email based system, one would not expect real-time control occurring through the resulting email network.

The small remote server code framework minimizes resulting system overhead, utilizing standard email servers to enable a capable, relatively stable remote platform with nearly zero administrative overhead. The remote server will periodically check for email on the server, POP the resulting message from the server, check its control syntax, and have the object code ready for programming. Recent FPAA devices now enable Floating-Gate (FG) device programming entirely on the device as an input data stream; therefore, the entire data stream, including $\mu \mathrm{P}$ code to execute programming, simply looks like a single stream of data to the system. Encapsulating this entire structure in a single file requires small, Unix-based code to communicate the file to be programmed. After the device is programmed and the input data is loaded into the processor, the IC proceeds to compute the resulting function, also storing data in local or in the remote server memory. The resulting output data results are pulled together and sent out by email to the host's chosen email address.

This approach enables programming by any small embedded devices (after design), particularly those with direct USB connections (tablet, phone) allowing minimal (Linux) code for programming and operation. Whether remote or not, these concepts allow an easy approach for networks of remote 
sensor nodes; for example, an IoT approach enabling real-time sensor processing that can send context aware results while requiring minimal support overhead for the approach.

Figure 4a shows the block diagram of the SoC FPAA used in this paper for experimental measurements [7]. This SoC FPAA enables nonvolatile digital and analog programmability through Floating-Gate (FG) devices, both in the routing fabric, but also for parameters for the computing elements. Figure 4a shows a typical FPAA block diagram that enables both analog and digital components in its Manhattan routing fabric, and most approaches for FPAA devices can be fitted into this resulting framework [11-16]. The blocks with simple digital components are called Computational Logic Blocks (CLB), and the blocks with analog and digital components are called Computational Analog Blocks (CAB). These devices enable useful computation out of using routing resources, such as Vector-Matrix Multiplcation (VMM) built out of the routing crossbar (i.e., floating-gate) switch matrices. These approaches are shown to generalize to building signal processing and classifier blocks [7].

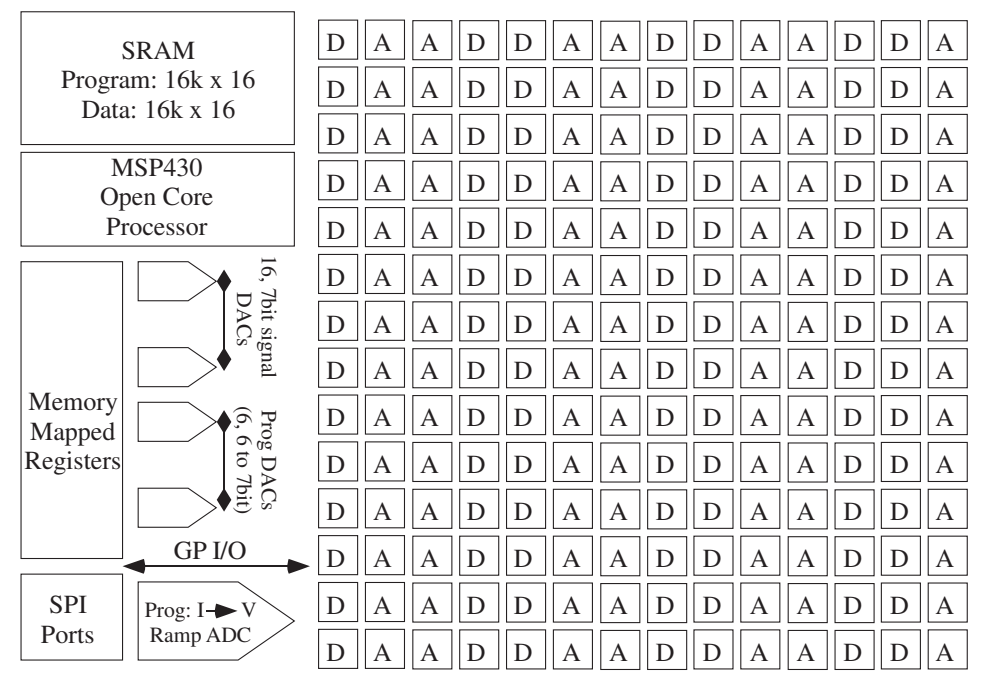

(a)

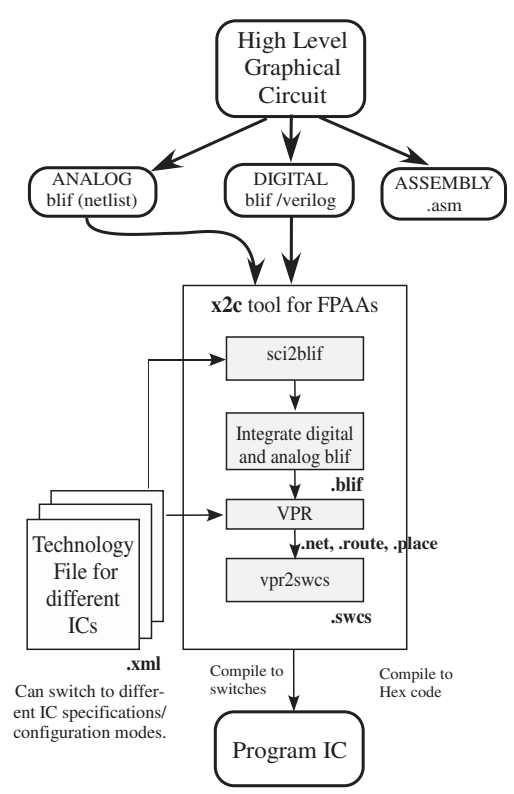

(b)

Figure 4. FPAA IC and Tool Infrastructure. (a) illustration of the structure of FPAA SoCs. The particular FPAA, or system of FPAAs and/or other components are defined by their different technology files, chosen by the user. Such an array could be a combination of components with analog (i.e., Computational Analog Block, or CAB) or digital components (i.e., Computational Logic Block, or CLB) that are connected through a switch matrix, as well as a range of I/O components and special additional components; (b) high level block diagram of the FPAA Xcos toolset, including the high level design environment as well as the compiler, $x 2 c$.

Figure $4 \mathrm{~b}$ illustrates the tool flow using a mixed-signal library of components. Large FPAA devices [7] require user friendly design tools to enable system design without requiring understanding of analog circuit components. This framework integrates a high-level design environment built in Scilab/X $\cos$ with a compilation tool, $x 2 c$, to compile from high level description to a targetable switch list to be programmed on the FPAA device. $x 2 \mathrm{c}$ is composed of sci2blif, and translates a block in scilab to .blif format, our modified VPR (Versatile Place and Route) tool [17], which acts as an input to vpr2swcs, as well as modified architecture files for analog-digital SoC. The graphical high level tool uses a palette for available blocks that compiles down to a combination of digital and analog hardware blocks, as well as software blocks on the resulting processor. Simply pressing one button in Ubuntu brings up the entire graphical working toolset. The approach allows a given user to access and use this 
toolset on their remote computing devices with minimal effort. This tool could be modified for other configurable structures, so the approach is wider than just FPAA devices.

Figure 5 shows FPAA IC board design, as well as systems using additional programmable components. The high-level graphical tool enables a user to be able to try different approaches to optimize the system performance, allowing consideration of tradeoffs of power, system utilization, time to market, etc. Digital Hardware-Software CoDesign is an established, although unsolved, discipline, starting from its early work [18]. Although engineers do design mixed mode systems, one still has many open questions of methodology in this field [19-21]. Including programmable and configurable analog computation to current digital approaches requires revisiting existing tradeoffs. The approach is focused on enabling system designers to integrate useful systems, while still enabling circuit experts to continue to develop creative and reusable designs within the same tool flow.

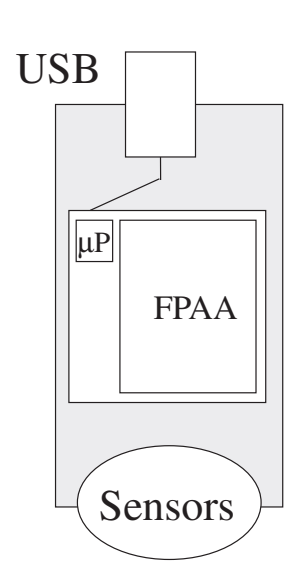

(a)

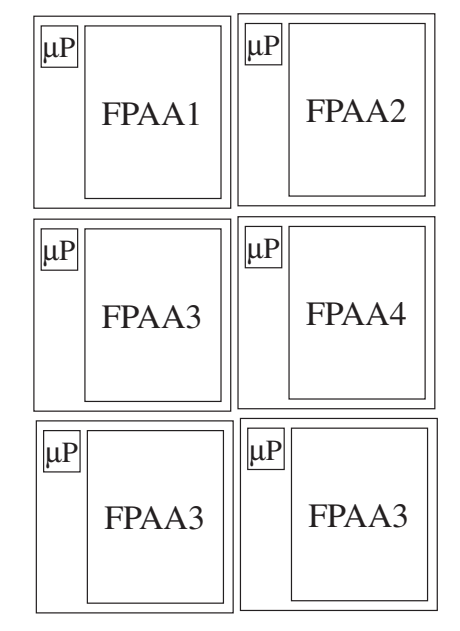

(b)

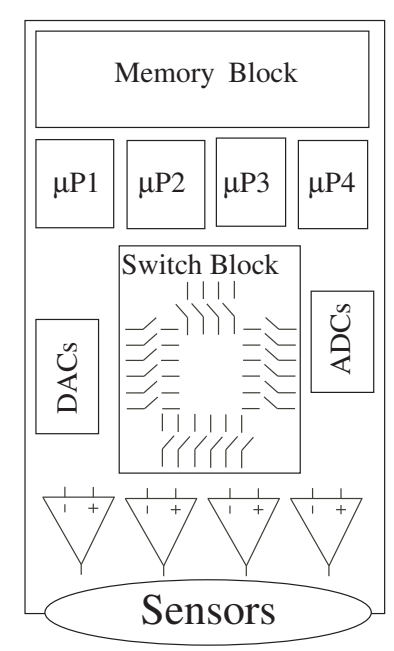

(c)

Figure 5. Possible approaches for mixed-mode computing systems. Implementation could be a (a) single FPAA device; (b) a board of FPAA devices; or even (c) a board with no FPAA devices but with programmable parameters and topology for a resulting board encoded in the resulting technology file.

\section{First Remote System Overview Example: Low Pass Filter}

Figure 6 shows an initial full tool example of the graphical interface and results for a first-order Low-Pass Filter (LPF) system, showing both simulation results and experimental results from the remote test system. Figures 7 and 10-12 show more complex tool examples using this remote system; we use this example to discuss the remote tool flow in detail. Xcos gives the user the ability to create, model, and simulate analog and digital designs [8]. The Xcos editor is standard blocks that are compartmentalized into classes or palettes that range from mathematical operations to digital signal processing. The editor allows the internal simulator to utilize the functionality of each block to compute the final answer. Our tool structure took advantage of user-defined blocks and palettes that can interact with Scilab inherent blocks. 


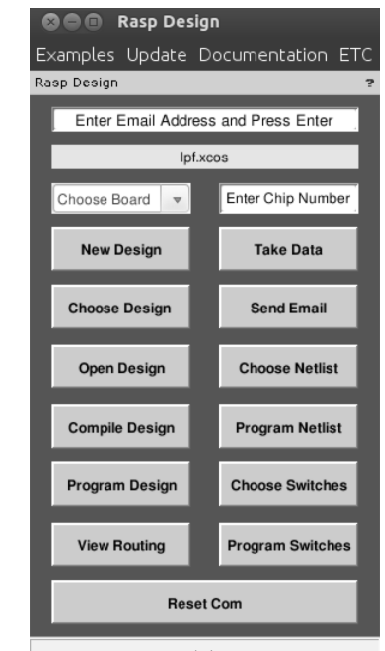

(a)

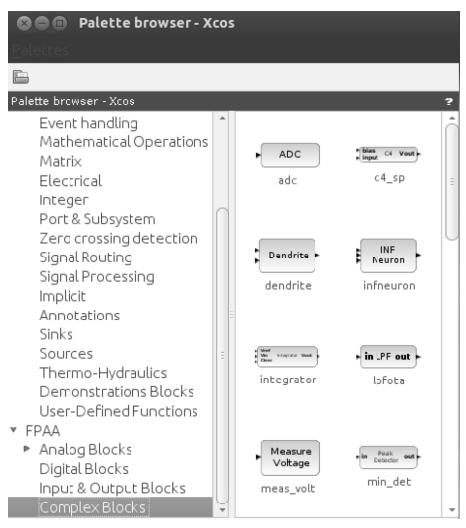

(b)

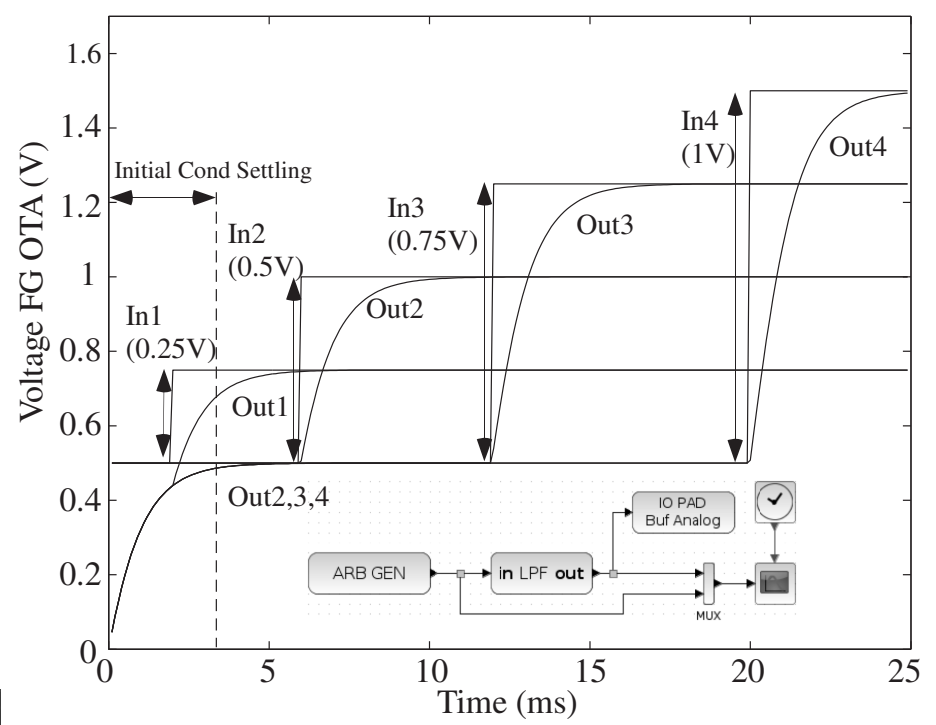

(c)

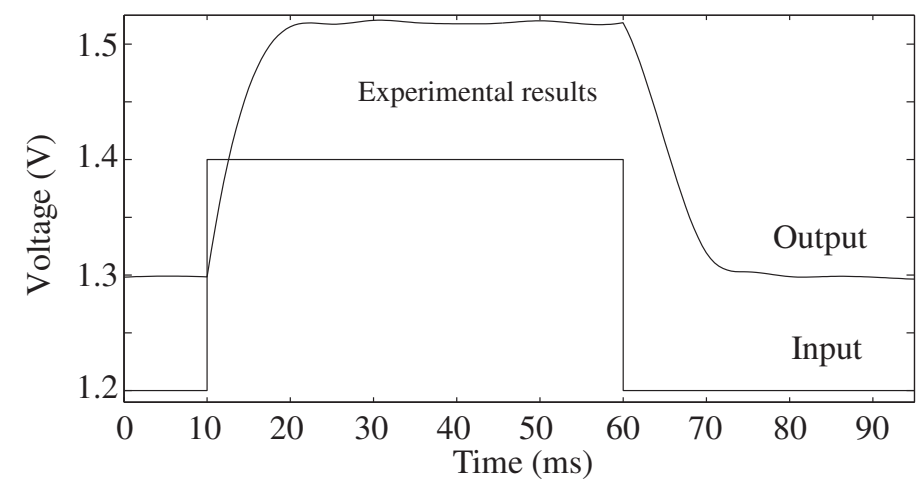

(d)

Figure 6. An example of the entire tool flow for a Low-Pass Filter (LPF) computation. (a) the user chooses basic design options through the FPAA Tools GUI, which starts running when the Scilab tools are started in the distributed Ubuntu Virtual Machine (VM); (b) snapshot of the Xcos palette for FPAA blocks. There are four sections, namely the Analog, Digital, Input/Output (I/O) and Complex Blocks; the Analog, Digital and I/O blocks consist of basic elements in different tiles of a chip. Complex blocks are pre-defined circuit blocks made of more than one basic element; (c) simulation results for four input and output computations. Lines and resulting blocks allow for vectorized as well as scalar inputs. Inset shows the Xcos diagram; the user sets parameters for simulation or for compiling into IC; and (d) experimental results for a one input and output computation.

Figure 6a shows the FPAA Tool GUI and grey buttons with the labels: New Design, Choose Design, Open Design, Compile Design, Program Design, and Take Data; these buttons open a new Xcos editor window, select a previously saved design, view the design in Xcos editor, convert the information in Xcos file into a programmable format, program hardware for the current Xcos file selected, and cause the hardware to be in the mode to take data, respectively. It includes a button which is labeled email, and a top box that one can put in an email address. These two small buttons on the tool framework show the minimal additional user overhead for using the remote system. 


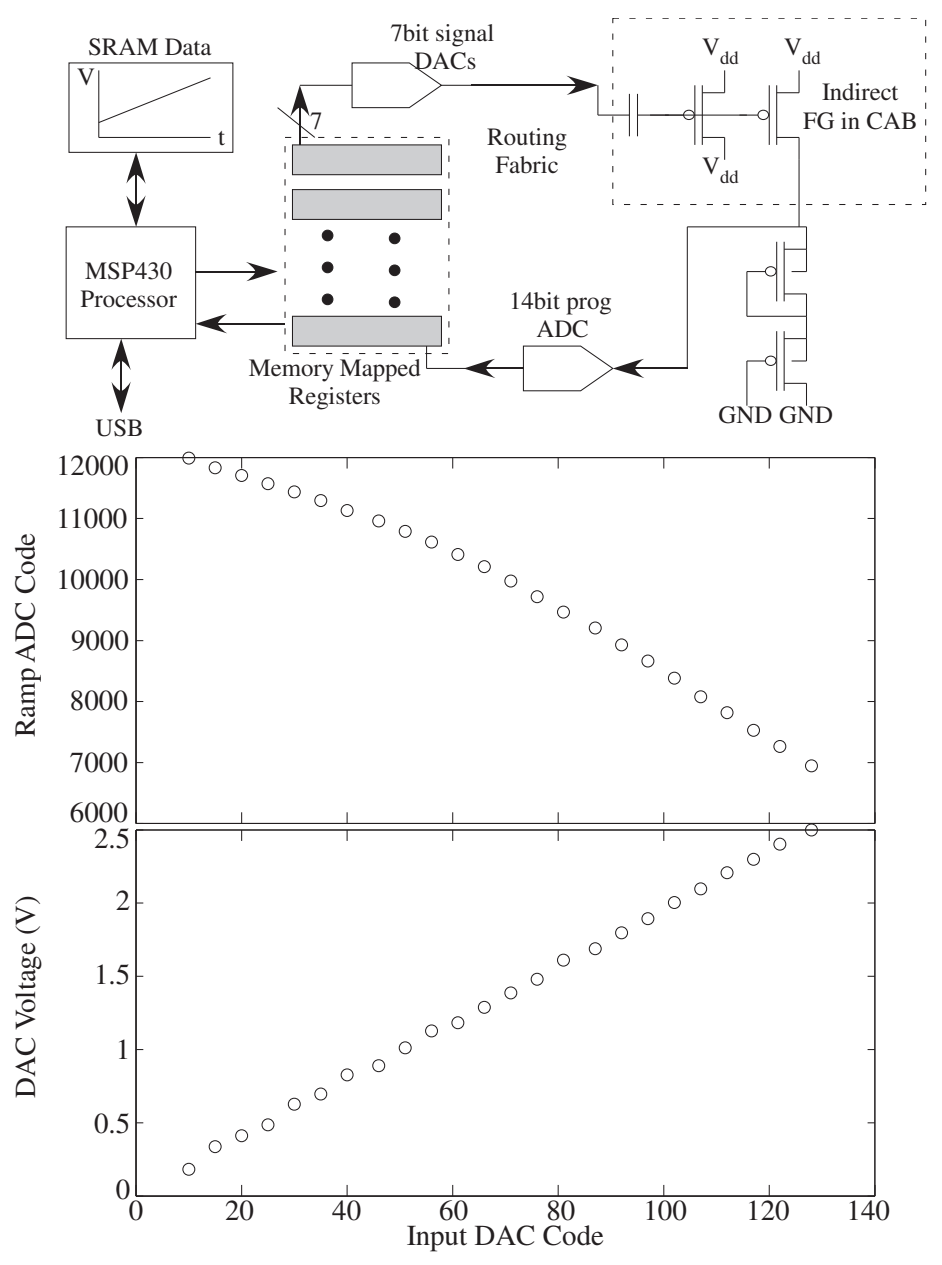

Figure 7. One basic voltage measurement scheme utilized in the SoC FPAA [7] for low-speed measurements utilizing the entire IC infrastructure. The $\mu \mathrm{P}$ design is an open-core MSP 430 processor with on-chip structures for seven-bit signal DACs, a ramp ADC, memory mapped General Purpose I/O (GPIO) and related components. The measurement through an FG transistor in a CAB utilizes the processor, signal DACs and memory mapped register, typical loops for instrumenting and measuring analog and digital blocks; Often, the FPAA computation utilizes all of these capabilities.

Our $\mathrm{X} \cos$ [22] tool uses user-defined blocks and libraries. When the user opens the $\mathrm{X} \cos$ editor, a palette browser is displayed, as shown in Figure 6b. The browser lists Scilab's collection of palettes as well as user defined palettes. One selects from a palette of available blocks to build the resulting system, which can be composed of a mixture of analog (blif), digital (verilog, blif), and software (assembly language) components. The Palette for FPAA Tools contains sub-palettes for blocks that are classified as analog, digital, inputs/outputs, and complex blocks. The Input/Output palette contains typical circuits like DACs, Arbitrary Waveform Generator, I/O pads, and Voltage Measurement. The digital palette contains typical circuits like a D Flip Flop and a clock divider. A few examples of complex blocks are an Low-Pass Filter (LPF), a Sigma-Delta ADC, and a VMM + Winner-Take-All (WTA) Classifier.

The single programming file, attached to the email for programming the remote system, is a compressed structure of multiple files. The tools output a single programming file, a combination of multiple files, that is used for FG programming and SRAM memory setup for the SoC FPAA [23]. The design tools can process the downloading of this file, as well as other devices (e.g., remote computer, tablet). The SoC FPAA devices now enable Floating-Gate (FG) device programming entirely on the device as an input data stream, therefore the entire data stream, including $\mu \mathrm{P}$ code to execute programming, simply looks like a single stream of data to the system. The FPAA utilizes an open-source 
$\mu \mathrm{P}$, embedded $16 \mathrm{k} \times 16$ SRAM for program and data memory, as well as the memory mapped registers for FG programming. We give the file definition in Table 1. The current system loads all of the data into the data SRAM $(16 \mathrm{k} \times 8)$ for this remote system. Currently, larger waveforms would require reloading the data SRAM and continued processing. We could compile an SPI port on the digital infrastructure and interface to the USB communication enabling streaming of larger data through USB. We expect this structure will remain stable across future generations; fortunately, each programming file is self contained for programming since it includes its own code and parameters for programming.

Table 1. Components of a Single Programming File.

\begin{tabular}{ccc}
\hline Function & Data Type & Core Files \\
\hline $\begin{array}{c}\text { erasing } \\
\text { and initialization }\end{array}$ & $\begin{array}{c}\text { Compiled } \\
\text { (assembly) }\end{array}$ & $\begin{array}{c}\text { tunnel_revtun_CAB.elf } \\
\text { switch_program.elf }\end{array}$ \\
\hline measuring outputs & Compiled & voltage_meas.elf \\
\hline input (e.g., DAC) data & data & input_vector \\
\hline FG block & data & output_info \\
\hline $\begin{array}{c}\text { info } \\
\text { (num, address) } \\
\text { switch list }\end{array}$ & data & $\begin{array}{c}\text { switch_info } \\
\text { target_info }\end{array}$ \\
\hline Course Prog $\mathrm{T}_{i n j}$ & data & pulse_width_table_offset_d1o2 \\
\hline Fine prog $\mathrm{V}_{d}$ table & data & Vd_table_30mV \\
\hline Program Memory & Compiled & prog_memory.elf \\
\hline Data Memory & data & input_vector \\
\hline
\end{tabular}

We explicitly kept the requirements for the computer as minimal as possible. We implemented this system running on 8 year old laptops running Ubuntu 12.04. These systems are considered too old for our computer support staff to allow on our network, and would be prohibited on the internal network. The basic requirements for this system as we have currently implemented the system include a flavor of Linux, a system capable of executing Python (3.4) script, that includes implementing an email POP server, a USB port to communicate with the FPAA board, and WiFi access to an open network. We also require email accounts for the communication between these blocks. Figure 8 shows the size of the three Python code modules required for this remote system. We expect these systems can be further reduced in complexity, both in code (Python) size and complexity. For example, one could easily imagine a device like a Raspberry Pi (e.g., Pi 3 versions with WiFi) [24], a device explicitly forbidden on our internal teaching and research networks. A Raspberry Zero with a wireless module attached could easily perform these operations.

\begin{tabular}{|c|c|c|c|c|}
\hline \multicolumn{3}{|c|}{ Size of Python Code } & \multicolumn{2}{|c|}{ Code Size of Representative Blocks } \\
\hline three parts: & Size & lines & Computational Block & $\mathrm{Siz}$ \\
\hline $\begin{array}{l}\text { get_email.py } \\
\text { program_fpaa.py } \\
\text { send_results.py }\end{array}$ & $\begin{array}{l}\text { 3Kbytes } \\
\text { 45.3Kbytes } \\
1.25 \text { Kbytes }\end{array}$ & $\begin{array}{c}82 \\
285 \\
47\end{array}$ & $\begin{array}{l}\text { Arb Waveform Generator + ADC } \\
\text { VMM + WTA XOR Classifier }\end{array}$ & $\begin{array}{c}\text { 3.1Kbyte } \\
73.8 \text { Kbytes }\end{array}$ \\
\hline
\end{tabular}

Figure 8. Memory sizes required for the Remote FPAA system, including the size of Python Code, broken down into its three fundamental modules; the size of compiled FPAA code for three representative systems. The Arb Waveform Generator + ADC includes a Signal DAC, FG FET, and ramp ADC. The VMM + WTA Exclusive OR (XOR) Classifier [25] includes 4 WTAs, Nmirrors, Shift register, 4 Signal DACs, FG FET, and ramp ADC. 


\section{Additional Configurable Remote Platform Considerations}

This approach gives a simple digital peripheral using a standard interface (i.e., USB), enabling a small IoT block interfaced through an email system to an open-source design/control tool. The resulting controlling device, whether it be directly connected through this digital port (i.e., phone or tablet) or through a network, can be a potentially simple OS enabling all features on the resulting system, including sensory/actuation devices connected to this remote platform.

Although the use case presented seems fairly simple, we believe this system is in the family of IoT devices,

"IoT is the network of physical objects that contain embedded technology to communicate and sense or interact with their internal states or the external environment."-[26]

This remote system fits this definition, particularly as it expands to larger number of systems and users.

"The IoT is significant because an object that can represent itself digitally becomes something greater than the object by itself." - [27]

Building a configurable mixed-mode system to have a standard USB and network interface enables these devices into the IoT space. Most IoT systems are not so small, or power constrained, but this system, coupled with sensors, integrates with this device and directly fits the IoT concept.

The authors chose a POP email system among the many alternatives for system communication. First, the simple structure of a POP email system (see for example [28]) fits with the limited operations required, is easy to code, and keeps the resulting computation requirements for operation as minimal as possible. POP supports simple download-and-delete, where this system only requires connection, message retrieval, and temporary storage (local). Anything more complex is extra overhead with little function. Email is ubiquitous, particularly for students. The wide variety of email services (e.g., Gmail) results in many painless options, without the need for server infrastructure. A simple POP server keeps the control code small allowing systems extending to small, remote platforms (e.g., devices worn on the body, etc.), particularly where low power battery operation is possible.

Second, starting from the inspiration of academic classroom experiences, the use of email protocol simplifies individual access while requiring effectively zero administrative support. For example, this remote system is being used in a graduate course this Spring 2016 [29]. This remote system approach allows a host system to even be operated anywhere, such as a coffee shop, without significantly affecting the system users. Most can get some form of a wireless network allowing basic email protocols on that network. Any administrative barrier for faculty to set up technology for their classes tends to result in the approach not ever being implemented. This remote system provides an alternate path through the constraints of the layers of resources and administration of those resources. We expect such a system can have significant research impact, and the ease of collaborators having shared systems opens up options for remote sensing and computing nodes with nearly zero required resource support.

Other remote test systems used in education typically require the user to remotely log-in to the system, and effectively take control of the system. From one of the classic circuit measurement systems [30], one sees attempts for remote test structure to replace a large number of dedicated lab stations with expensive equipment, similar to the setups used at Caltech CNS 182 (CNS 182: Analog VLSI and Neural Systems course taught at Caltech between 1983 and 1996, using the textbook by the same name [31]. The course no longer exists, and nothing is hosted on Caltech sites any longer.), and later at Georgia Tech (GT) utilizing similar dedicated lab stations that transformed into the earliest FPAA devices [29]. Additional remote systems have attempted other concepts having users log-in and control a remote computer [32-35], as well as debates over the right use of remote hardware for classes [32]. These approaches involve using a hardware board (e.g., Spartan 3 or Arduino board) and using graphical languages like Labview (e.g., [36]). Much of the time on the system relates to the user 
thinking about their problem, rather than performing operations on their experiment; this paper's system gets around these issues while using a tool approach identical when a physical board is used.

This remote system approach is different from the area of one-way updating FPGA software for a device in the field. Remote FPGA reconfiguration already utilizes internet connections on allowed networks (with any resulting permission overhead), and can update FPGAs using protocol like User Datagram Protocol (UDP) [37]. Xlinix provides direct developer support when using external devices (e.g., CPLD) [9], or even cases using the FPGA as part of the updating infrastructure [10]. Hardware systems are designed for in-field FPGA updating [38], including space applications [39]. This paper's remote system looks towards an expanded purpose, while also not being subjected to any network infrastructure constraints. This system may in fact be used by the authors for updating of FPAA and other configurable devices in the future, although this application is not our driving motivation.

Security management was not implemented in this first system implementation, but is a focus area going forward. One would want a form of authorization (i.e., a user access code) as part of the email message. Using a reduced email based system does reduce a significant amount of potential security issues compared to a web-based system, as well as not needing to support a wide range of internet browsers. The Linux based system and email retrieval system is used to download files, extract resulting targeting files, and use those files for targeting; we do not use an entire email browser for the resulting system, eliminating many potential virus issues. The biggest security issue seems to be uncompressing the resulting files, where that is the only place we need to execute anything on the files, and should be developed as a trusted source. We also expect a host of issues will arise as people work with such a system where outside groups, say running the remote system, might have exposure to the user's designs, and resulting Intellectual Property. We expect current approaches towards individual security will be used where applicable.

\section{The Range of User Interfacing Expands Remote User Capability}

Figure 9 shows that the remote server was partially enabled by a simple digital (USB) interface, with simple interfacing between the FPAA IC (or multiple ICs) to the resulting USB infrastructure to the host device. The input data, output data, FG programming, and other control functions all move through a single standard digital USB interface; the device to the remote system looks like any other embedded, USB peripheral, where the tools handle a similar case whether the board is local to the tools or emailed to the remote server. The user needs to understand both the analog and digital capability of the FPAA IC, and the resulting input and output blocks for the system as well as the resulting system control, at the level allowed for the high-level tool framework (Xcos/Scilab). A user will often use digital (GPIO) or analog (DAC through GPIO) input blocks, interfaced through the SRAM memory and $\mu \mathrm{P}$ control, and will often use digital as well as analog compiled ADC through GPIO or Vmeasure through slower and more accurate 14-bit ADC. The tool framework compiles down the resulting analog, digital, and $\mu \mathrm{P}$ components, as well as the vector input into the system. The setup uses no external pins; one could connect multiple devices, heterogeneous devices, and additional sensors, but to the external world, the device is still a simple USB connected device. The USB interface is connected through serial interfaces, using a simple serial (8n1) interface through an FTDI 2232D IC (FTDI, Glasgow, Scotland) on the board.

Figure 9 shows the high-level blocks representation, similar to the corresponding Xcos diagram, both including computational blocks, as well as input and output blocks available and their interfacing into the $\mu \mathrm{P} / \mathrm{SRAM}$ memory block. The arbitrary waveform generator connects data to a vector input representation versus time in the Scilab workspace that gets converted, where needed (i.e., DAC devices), as well as packed with the programming file; the interface for the data is directly set by the high-level tools. A short list of potential on-chip interfacing options for moving data in and out of the $\mu \mathrm{P} /$ SRAM memory are: 
- General Purpose Input/Output (GPIO) memory mapped digital registers;

- Hardware interrupts based on routed fabric signals;

- Signal DAC through memory mapped registers;

- Compiled ADC coupled with FG elements in the fabric through GPIO registers.

These approaches are all voltage-mode inputs and outputs, consistent with level $=1$ block definition for system building [40]. Furthermore, blocks can have some assembly code (or completely assembly code) as part of the functionality, and, therefore, would interact with memory, potentially, more directly. Where necessary, one can measure currents through compiled transimpedance amplifiers, switched capacitor networks, and related circuit techniques. One key constraint on any algorithm is efficiently using the $16 \mathrm{k} \times 8$ SRAM data space either to hold data, or buffer data coming through the serial communication from the host system running the remote interface.
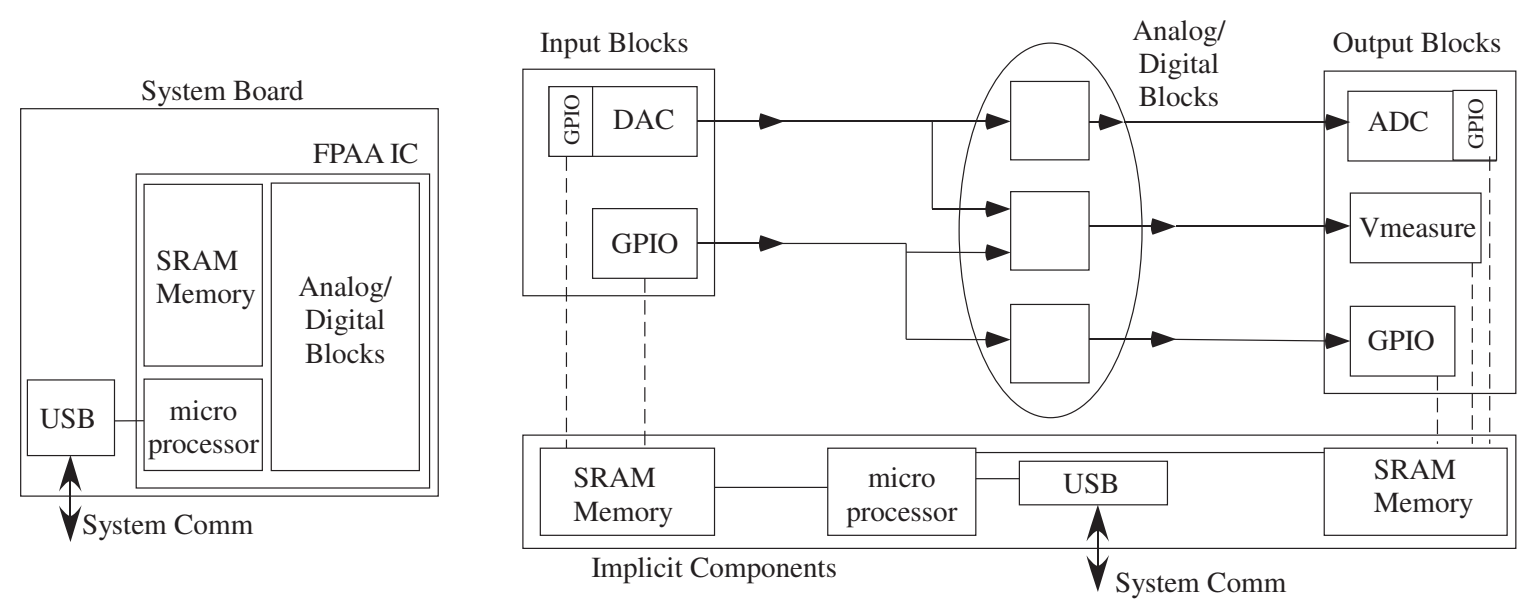

Figure 9. Our approach is enabled through a single port of communication, through a USB port, to a self contained programmable and configurable mixed-signal IC through its programmable interface. The entire IC is the computing system, and all components are part of the computation.

The availability of ADC blocks determines the particular computing architecture. Figure 10 shows a compiled ramp ADC in the FPAA fabric; one would want a wide library of FPAA compilable ADCs using the FG device density. A pipelined one-bit ADC architecture would be the lowest practical latency because computation requiring smaller latencies would be done in analog. These devices are used mostly for acquisition of data or circuit debugging. Algorithmic converters, being related to the pipelined ADCs, would most likely win over successive-approximation ADCs because they do not require designing a separate DAC for the system.

Figure 11 shows a simple compiled ramp ADC converter to illustrate the flow from input SRAM memory data to the computed output SRAM memory data. The resulting simple 8-bit ramp ADC illustrates using the digital infrastructure and $\mu \mathrm{P}$ to move analog signals into stored SRAM memory. The simplicity only requires part of a single $\mathrm{CAB}$ element while still giving reasonable monotonic performance with some curvature due to the nonideality of the current source element creating the ramp; Measurement examples use 4k Samples per Second (SPS) ADC sampling.

Figure 11 shows a complete computing loop for data through a board used as a remote test. The initial data is loaded into SRAM as part of the programming structure, processed through one of multiple memory mapped seven-bit DACs, processed through a first-order LPF block programmed with a corner frequency of $150 \mathrm{~Hz}$, and measured through the above ADC block, arriving back as stored solution vectors in SRAM that are transmitted back to the user. The LPF block is the programmable Transconductance Amplifier (TA) with the resulting routing capacitance, as used in the previous section; $\mathrm{G}_{m}-\mathrm{C}$ filter topologies utilize line capacitance setting the filter corner by the transconductance 
of the OTA amplifier. This compiled ADC integrates the compare + ramp functions compile into a single $C A B$, routing the digital output bit to GPIO. The ADC polls for the digital bit to change while counting in the processor; more advanced blocks utilize interrupts and compiled counter blocks in the CLBs for the conversion. The ADC is not meant to be the highest precision component, but a very small, monotonic ADC calibrated for the resulting response as necessary.

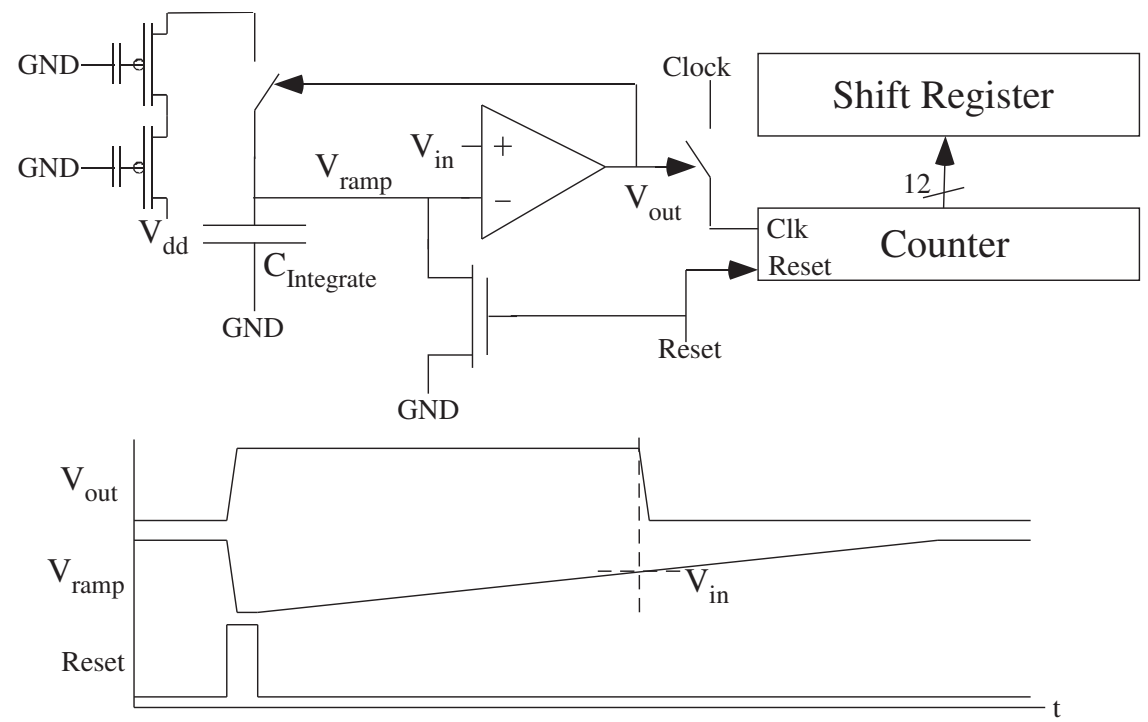

Figure 10. For the remote system, one key aspect is the range of potential methods for voltage measurement in the fabric to connect to the $\mu \mathrm{P}$ device. Typically, these devices include direct digital inputs, with resulting level comparisons, ADC, as well as a range of other classifier components. One can compile a Ramp ADC in the routing fabric to operate at a range of sample frequencies and resolution.
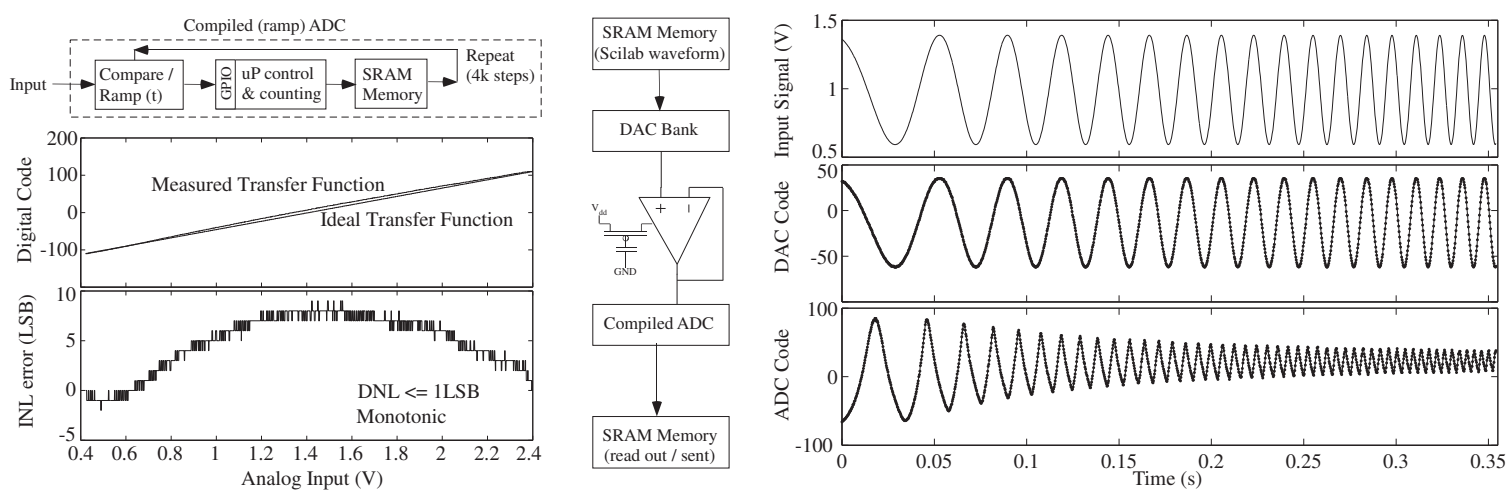

Figure 11. Measurement of an LPF frequency response. The programmed corner frequency of the LPF (the TA and routing capacitance) is $150 \mathrm{~Hz}$. When the experiment is programmed, components in the $\mu \mathrm{P}$, digital, and analog devices are used. The resulting device is measured by applying a linear chirp input signal going from $25 \mathrm{~Hz}$ to $250 \mathrm{~Hz}$, showing the resulting signal attenuation, as expected, as well as the resulting signals from the input seven-bit DAC as well as the eight-bit ramp-ADC (4kSPS). The compiled eight-bit ramp 4kSPS ADC polls for the digital bit to change while counting in the processor. The digital bits are routed through GPIO. The output of the ADC inverts the resulting response from the original signal. 
At this stage, one might ask how the system handles users or student bugs in their compiled code. The system allows for bugs, as well as debugging of the system. At one level, the system operates as programmed, particularly all of the hardware components. The input data will be applied, and the output data will be read and emailed to the user. Like debugging other software systems, the output results will potentially show the result of these bugs. The student/user will interpret the results, similar to other software systems, and modify their design accordingly. Furthermore, one could imagine bugs in the $\mu \mathrm{P}$ code that would hang the system. In this case, if the system takes too long to run (like $1 \mathrm{~min}$ ), the controlling system halts the process, emails those results, and the system proceeds to the next item in the queue.

Figure 12 shows a more complex example using the remote FPAA system, a parallel bank of bandpass filters and amplitude detection typically used for low-power sub band analysis, as another example of co-design between assembly, analog, and digital components and interfacing. The corner frequencies are programmed between $100 \mathrm{~Hz}$ and $750 \mathrm{~Hz}$, seen by the different peaks in the chirp response in Figure 12. This example uses the same SRAM memory, seven-bit input DAC, and compiled ADC. A similar chirp signal linearly varies between 25 and $1 \mathrm{kHz}$.

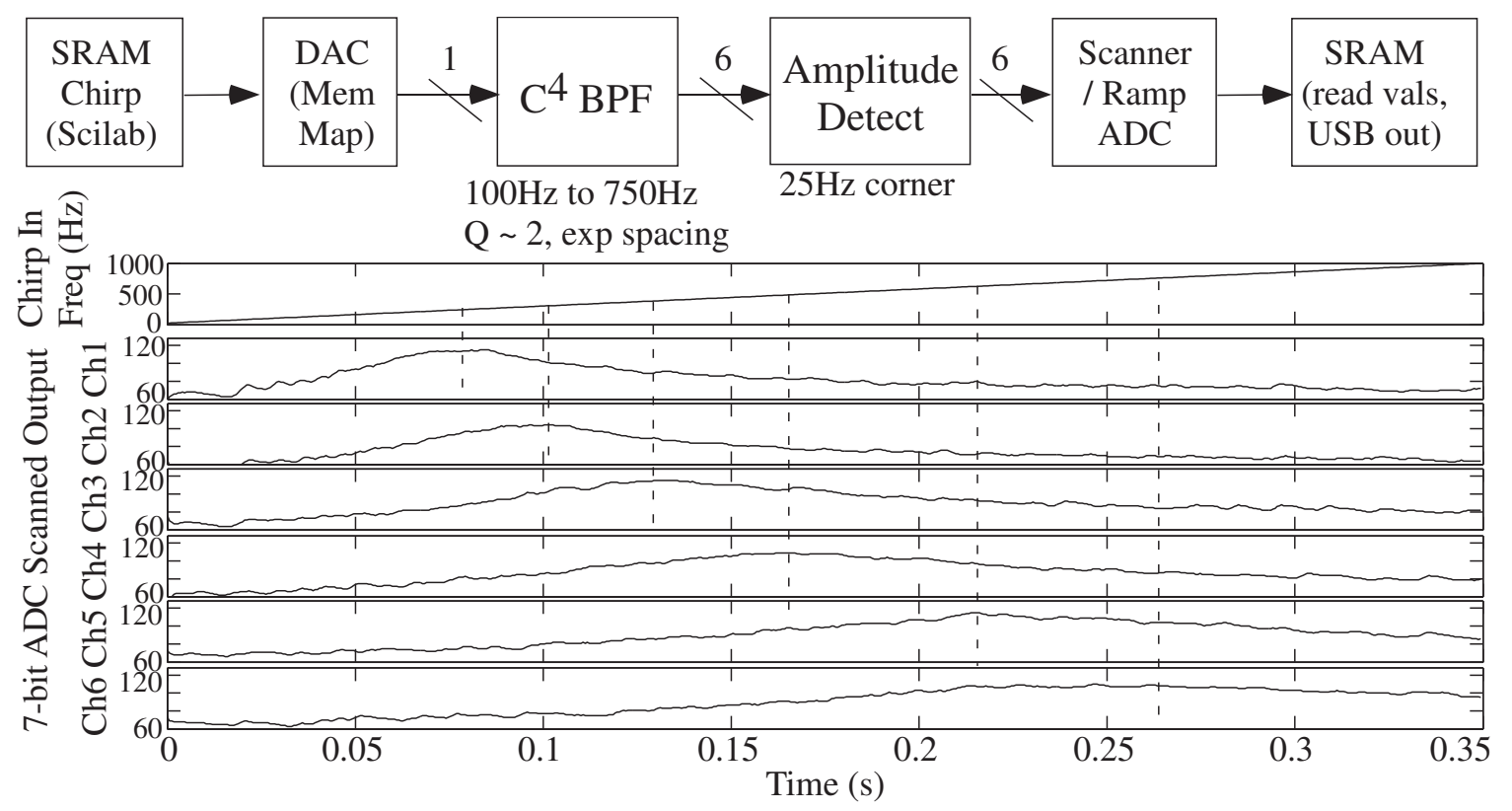

Figure 12. Block diagram (similar to Scilab/Xcos definition) and output results for a bank of six BandPass Filter (BPF) and Amplitude detect elements compiled and measured through a remote FPAA board. The input chirp signal, a linear sweep between $25 \mathrm{~Hz}$ and $1 \mathrm{kHz}$, is stored on-chip SRAM to be played through a memory-mapped DAC. The output signals went through a demultiplexing module, compiled using the shift-registers located in routing fabric, and then through the same eight-bit ramp ADC module; this measurement only used the upper half of the positive values (effectively six-bit). The linear frequency sweep with time is illustrated, as well as the resulting outputs of all six frequency channels, each programmed to a different frequency location (exponentially spaced).

\section{Conclusions}

We presented a novel remote test system, enabled by configurable analog-digital ICs to create a simple interface for a wide range of experiments. Our remote test system requires no additional setup, resulting both from using highly configurable FPAA devices, as well as from the advancement of straight-forward digital interfaces for the resulting experimental FPAA system. An analog-digital programmable and configurable IC system, enabled by FPAA device(s), requiring a single digital external digital interface opens opportunities for a simple remote test infrastructure. The system 
overhead requirements are straightforward, requiring simple email handling, available over almost all network systems with no additional requirements. We plan to release the open-source code for the remote system development after this journal paper is in press.

Opportunities appear both in academic, as well as research and industrial applications. This technical platform enables collaborators in different areas to investigate items on a single platform; setting up a system is straight-forward and capable for multiple systems. These approaches have been utilized as part of a graduate level class at GT [29,41]. Our novel open-source tool platform empowers the user to do seamless low-power analog-digital CoDesign in a single environment. Our FPAA SoCs consist of an integrated processor, I/O peripherals, and an FPAA comprised of analog and digital components [7], although the approaches can be extended to other platforms. Our approach integrates multiple open-source tools, using Scilab and VPR, to develop a coherent user-friendly design flow, with a custom software toolkit that generates and implements high-level simulation and experimental measurement of the resulting hardware system [8]. The ability to seamlessly move between tools designed for a board in hand as well as a remote test system has greatly improved the student's interest in using either hardware platform, as well as keeping development complexity for such a complex laboratory course under control.

Acknowledgments: The authors would like to thank Mark Smith, KTH (Kista, Sweeden) for the original inspiration to build an email-based remote system FPAA system.

Author Contributions: Jennifer Hasler: Developed the original concept, guided this development, co-developed the SoC development structure, and wrote this paper. She has co-taught multiple courses utilized this remote system. Sahil Shah: Developed the infrastructure examples, SoC development structure, and co-manager of the early remote system examples. He co-taught a course utilizing this remote system. Sihwan Kim: Co-developer of the core python code, and co-manager of the early remote system examples. He co-taught a course which utilizing this remote system. Ishan Kumal Lal: Co-developer of the core python code.

Conflicts of Interest: The authors declare no conflict of interest.

\section{References}

1. Maiti, A.; Maxwell, A.D.; Kist, A.A.; Orwin, L. Merging Remote Laboratories and Enquiry-based Learning for STEM Education. Int. J. Online Eng. 2014, 10, 50-57.

2. Harward, V.J.; del Alamo, J.A.; Lerman, S.R.; Bailey, P.H.; Carpenter, J.; DeLong, K.; Felknor, C.; Hardison, J.; Harrison, B.; Jabbour, I.; et al. The iLab Shared Architecture: AWeb Services Infrastructure to Build Communities of Internet Accessible Laboratories. Proc. IEEE 2008, 96, 931-950.

3. Lowe, D.; Murray, S.; Lindsay, E.; Liu, D. Evolving Remote Laboratory Architectures to Leverage Emerging Internet Technologies. IEEE Trans. Learn. Technol. 2009, 2, 289-294.

4. Suosa, N.; Alves, G.R.; Gericota, M.G. An Integrated Reusable Remote Laboratory to Complement Electronics Teaching. IEEE Trans. Learn. Technol. 2010, 3, 265-271.

5. Bochicchio, M.A.; Longo, A. Hands-on Remote Labs: Collaborative Web Laboratories as a Case Study for IT Engineering Classes. IEEE Trans. Learn. Technol. 2009, 2, doi:10.1109/TLT.2009.30.

6. Cooper, M.; Ferreira, J.M.M. Remote Laboratories Extending Access to Science and Engineering Curricular. IEEE Trans. Learn. Technol. 2009, 2, 342-353.

7. George, S.; Kim, S.; Shah, S.; Hasler, J.; Collins, M.; Adil, F.; Wunderlich, R.; Nease, S.; Ramakrishnan, S. A Programmable and Configurable Mixed-Mode FPAA SOC. IEEE Trans. VLSI 2016, 24, 2253-2261.

8. Collins, M.; Hasler, J.; George, S. An Open-Source Toolset Enabling Analog-Digital-Software Codesign. J. Low Power Electron. Appl. 2016, 6, 3, doi:10.3390/jlpea6010003.

9. Park, K.; Kim, H. Remote FPGA Reconfiguration Using MicroBlaze or PowerPC Processors. XAPP441 (v1.1), 9 September 2006. Available online: http://www.xilinx.com/support/documentation/application_notes/ xapp441.pdf (accessed on 8 June 2016).

10. Kuramoto, R. QuickBoot Method for FPGA Design Remote Update. XAPP1081 (v1.3), 18 March 2014. Available online: http://www.xilinx.com/support/documentation/application_notes/xapp1081.pdf (accessed on 8 June 2016). 
11. Basu, A.; Brink, S.; Schlottmann, C.; Ramakrishnan, S.; Petre, C.; Koziol, S.; Baskaya, F.; Twigg, C.; Hasler, P. A Floating-Gate-Based Field Programmable Analog Array. IEEE JSSC 2010, 45, 1781-1794.

12. Becker, J.; Manoli, Y. A continuous-time field programmable analog array (FPAA) consisting of digitally reconfigurable G M-cells. IEEE ISCAS 2004, 1, doi:10.1109/ISCAS.2004.1328389.

13. Cowan, G.; Melville, R.; Tsividis, Y. A VLSI Analog Computer/math Co-Processor for a Digital Computer; Columbia University: New York, NY, USA, 2005.

14. Lajevardi, P.; Chandrakasan, A.P.; Lee, H.-S. Zero-crossing detector based reconfigurable analog system. JSSC 2011, 46, 2478-2487.

15. Wunderlich, R.B.; Adil, F.; Hasler, P. Floating gate-based field programmable mixed-signal array. IEEE TVLSI 2013, 21, 1496-1505.

16. Rumberg, B.; Graham, D.W. Reconfiguration Costs in Analog Sensor Interfaces for Wireless Sensing Applications. In Proceedings of the 2013 IEEE 56th International Midwest Symposium on Circuits and Systems (MWSCAS), Columbus, OH, USA, 4-7 August 2013; pp. 321-324.

17. Luu, J.; Goeders, J.; Wainberg, M.; Somerville, A.; Yu, T.; Nasartschuk, K.; Nasr, M.; Wang, S.; Liu, T.; Ahmed, N.; et al. VTR 7.0: Next Generation Architecture and CAD System for FPGAs. ACM Trans. Reconfigurable Technol. Syst. 2014, 7, 6:1-6:30.

18. Wolf, W. Hardware-software co-design of embedded systems. Proc. IEEE 1994, 967-989.

19. Jerraya, A.A.; Wolf, W. Hardware/Software Interface Codesign for Embedded Systems. IEEE Comput. 2005, 63-69.

20. Teich, J. Hardware/Software Codesign: The Past, the Present, and Predicting the Future. Proc. IEEE 2012, 100, 1411-1430.

21. Sampson, A.; Bornholt, J.; Ceze, L. Hardware-Software Co-Design: Not Just a Cliché; Advances in Programming Languages (SNAPL-15); Leibniz-Zentrum für Informatik: Wadern, Germany, 2015; pp. 262-273.

22. Scilab Enterprises. Scilab: Free and Open Source Software for Numerical Computation; Scilab Enterprises: Orsay, France, 2012.

23. Kim, S.; Hasler, J.; George, S. Integrated Floating-Gate Programming Environment for System-Level Ics. IEEE Trans. VLSI 2016, 24, 2244-2252.

24. Raspberry Pi-Teach, Learn, and Make with Raspberry Pi. Available online: https://www.raspberrypi.org/ (accessed on 8 June 2016).

25. Ramakrishnan, S.; Hasler, J. The VMM and WTA as an analog classifier. IEEE Trans. VLSI 2014, 22, 353-361.

26. Internet of Things. Available online: http://www.gartner.com/it-glossary/internet-of-things/ (accessed on 8 June 2016).

27. Internet of Things (IoT). Available online: https://www.techopedia.com/definition/28247/ internet-of-things-iot (accessed on 8 June 2016).

28. Dean, T. Network+ Guide to Networks, 5th ed.; Course Technology Cengage Learning: Boston, MA, USA, 2010; p. 519.

29. Hasler, J.; Kim, S.; Shah, S.; Adil, F.; Collins, M.; Koziol, S.; Nease, S. Transforming Mixed-Signal Circuits Class through SoC FPAA IC, PCB, and Toolset. In Proceedings of the European Workshop on Microelectronics Education, Southampton, UK, 11-13 May 2016.

30. Knight, C.D.; DeWeerth, S.P. A shared remote testing environment for engineering education. In Proceedings of the 26th Annual Conference, Frontiers in Education Conference, Salt Lake City, UT, USA, 6-9 November 1996; Volume 3, pp. 1003-1006; doi:10.1109/FIE.1996.567661.

31. Mead, C. Analog VLSI and Neural Systems; Addison Wesley: Boston, MA, USA, 1989.

32. Poindexter, S.E.; Heck, B.S. Using the web in your courses: What can you do? What should you do? IEEE Control Syst. Mag. 1999, 19, 83-92.

33. Esche, S.K.; Prasad, M.G.; Chassapis, C. Remotely Accessible Laboratory Approach For Undergraduate Education. AGE 2000, 5, 5.525.1-5.525.10.

34. Casini, M.; Prattichizzo, D.; Vicino, A. E-Learning by Remote Laboratories: A New Tool for Control Education. In Advances in Control Education (ACE 2003), Proceedings of the volume from the 6th IFAC Symposium, Oulu, Finland, 16-18 June 2003; pp. 73-78.

35. Saleheen, F.; Giorgi, S.; Smith, Z.; Picone, J.; Won, C.H. Design and Evaluation of a Web-Based Virtual Open Laboratory Teaching Assistant (VOLTA) for Circuits Laboratory; American Society for Engineering Education: Washington, DC, USA, 2015. 
36. Karthik, S.; Shreya, P.; Srihari, P.; Viswanath, N.M. Remote Field Programmable Gate Array (FPGA) Lab. Int. J. Res. Eng. Technol. 2014, 3, 842-845.

37. Kurose, J.F.; Ross, K.W. Computer Networking: A Top-Down Approach; Pearson Education: Boston, MA, USA, 2010; ISBN 978-0-13-136548-3.

38. Vliegen, J.; Mentens, N.; Verbauwhede, I. A Single-chip Solution for the Secure Remote Configuration of FPGAs using Bitstream Compression. In Proceedings of the IEEE Reconfigurable Computing and FPGAs (ReConFig), Cancun, Mexico, 9-11 December 2013; pp. 1-6, doi:10.1109/ReConFig.2013.6732330.

39. Surratt, M.; Loomis, H.H.; Ross, A.A.; Duren, R. Challenges of Remote FPGA Configuration for Space Applications. In Proceedings of the IEEE Aerospace Conference, Big Sky, MT, USA, 5-12 March 2005; pp. 1-9, doi:10.1109/AERO.2005.1559549.

40. Schlottmann, C.R.; Hasler, J. High-Level Modeling of Analog Computational Elements for Signal Processing Applications. IEEE Trans. VLSI 2014, 22, 1945-1953.

41. Collins, M.; Hasler, J.; George, S. Analog systems education: An integrated toolset and FPAA SoC boards. In Proceedings of the 2015 IEEE International Conference on Microelectronics Systems Education (MSE), Pittsburgh, PA, USA, 20-21 May 2015; pp. 32-35.

(C) 2016 by the authors; licensee MDPI, Basel, Switzerland. This article is an open access article distributed under the terms and conditions of the Creative Commons Attribution (CC-BY) license (http://creativecommons.org/licenses/by/4.0/). 